\title{
PLANE PEANIAN CONTINUA WITH UNIQUE MAPS ON THE SPHERE AND IN THE PLANE*
}

\author{
BY \\ V. W. ADKISSON

\section{INTRODUCTION}

The plane peanian continuum $\dagger M$ is said to have a unique map on a spherical surface (or a plane) if, and only if, for any topological image $M^{\prime}$ of $M$ on a sphere (or plane) $S^{\prime}$ and any topological image $M^{\prime \prime}$ of $M$ on a sphere (or plane) $S^{\prime \prime}$, every homeomorphism of $M^{\prime}$ into $M^{\prime \prime}$ can be extended to a homeomorphism of $S^{\prime}$ into $S^{\prime \prime}$.

It is the purpose of this paper to characterize the plane peanian continua that have unique maps on the sphere or in the plane. $\ddagger$

Definitions. The simple closed curve $J$ of a cyclicly connected continuum $C$ is called a bounding circuit of $C$ provided that for any two maximal connected components $H$ and $K$ of $C-J$ the sets $\bar{H} \cdot J$ and $\bar{K} \cdot J$ lie respectively on two distinct $\operatorname{arcs} A X B$ and $A Y B$ of $J$.§

A split circuit is a bounding circuit $J$ such that $C-J$ contains at least two components.

If $C$ is cyclicly connected, then $C$ is triply connected if, and only if, it is impossible to express $C$ as the sum of two closed connected sets $A$ and $B$ such that neither $A$ nor $B$ is an arc and the set $A \cdot B$ consists of two distinct points of $C$.\|

Theorems IV and V state the principal results of this paper.

THEOREM IV. The plane peanian continuum $M$ has a unique map on the sphere if, and only if, one of the following conditions holds:

* Presented to the Society, September 9, 1937; received by the editors July 22, 1937.

$\dagger$ A plane peanian continuum is a peanian continuum (continuous curve) that has a map (topological image) in the plane or on the sphere. For a characterization of these continua see W. S. Claytor, Topological immersion of peanian continua in a spherical surface, Annals of Mathematics, vol. 35 (1934), pp. 809-835. See also Claytor, Peanian continua not imbeddable in a spherical surface, ibid., vol. 38 (1937), pp. 631-646.

$\ddagger$ This problem was suggested by J. R. Kline. The author also wishes to express his appreciation of suggestions by Saunders MacLane which have led to improvements in this paper.

$\S$ For cyclicly connected continua bounding circuit is equivalent to boundary curve as defined by Claytor, loc. cit., first paper, p. 809. A simple closed curve $J$ of $M$ is called a boundary curve of $M$ provided that there do not exist in $M-J$ distinct components $H$ and $K$ such that (1) a point pair of $\bar{H} \cdot J$ separates a point pair of $\bar{K} \cdot J$ on $J$, or (2) $\bar{H} \cdot J=\bar{K} \cdot J=$ three distinct points. Both definitions will be found useful in later proofs.

\| Cf. definition of triply connected graphs, H. Whitney, Congruent graphs and the connectivity of graphs, American Journal of Mathematics, vol. 54 (1932), p. 158. 
(1) $M$ is acyclic and consists of either a simple arc or a triod.*

(2) $M$ contains one cyclic element $C \dagger$ which is a maximal triply connected cyclic curve of $M$, and $M-C$ consists of at most a countable number of arcs, $a_{1}, a_{2}, a_{3}, \cdots$, such that $\bar{a}_{i} \cdot \bar{a}_{j}=0,(i \neq j)$, and each $\bar{a}_{i} \cdot C$ is a single point which lies on only one bounding circuit of $C, \ddagger$ provided that if $C$ is a simple closed curve, then $M-C$ is at most a simple arc.

THEOREM V. The plane bounded peanian continuum $M$ has a unique map in the plane if, and only if, $M$ is one of the following curves:

(1) a simple arc,

(2) a triod,

(3) a simple closed curve,

(4) a curve $M$ such that $M$ contains a closed 2-cell $C$ and $M-C$ consists of at most a countable number of arcs $a_{1}, a_{2}, a_{3}, \cdots$, such that $\bar{a}_{i} \cdot \bar{a}_{j}=0,(i \neq j)$, and each $\bar{a}_{i} \cdot C$ is a single point which lies on the only bounding circuit of $C . \S$

\section{Preliminary Theorems}

Theоrem I. The plane cyclic (cyclicly connected) peanian continuum $C$ is triply connected if, and only if, $C$ contains no split circuit.

THEOREM II. The plane cyclic peanian continuum $C$ has a unique map on the sphere if, and only if, $C$ is triply connected.

COROLlaRY 1. If $J$ is a bounding circuit of $C$ which is not a split circuit, then in every map of $C$ on the sphere (or plane) the image of $J$ is the boundary of a complementary domain of the map of $C$.

COROLLARY 2. If $J$ is a split circuit of $C$ there is some map of $C$ on the sphere (or plane) in which the image of $J$ is the boundary of a complementary domain of the map of $C$.\|

Definition. The point $p$ of $M$ is a split-point of $M$, if and only if, $M$ can be expressed as the sum of two closed connected sets $A$ and $B$ such that $A \cdot B=p$, and such that if $A$ or $B$ is an arc, then $p$ is not an end point of that arc.

THEOREM III. If the plane peanian continuum $M$ has a unique map on the sphere or in the plane, then $M$ does not contain a split-point.

* Three $\operatorname{arcs} P A, P B, P C$ with $P$, and only $P$, common to any two.

$\dagger \mathrm{G}$. T. Whyburn, Concerning the structure of a continuous curve, American Journal of Mathematics, vol. 50 (1928), p. 168.

$\ddagger$ Each point $\bar{a}_{i} \cdot C$ is a non-local cut-point of $C$. See Theorem VI and the alternative statement of Theorem IV at the end of this paper.

$\S$ See Claytor. loc. cit., p. 810, Corollary (C).

|| This is a generalization of Proposition K, Claytor, loc. cit., first paper, p. 828. 


\section{Proofs of Theorems I-V}

Proof of Theorem I. Let $C$ be a map of the given curve on a plane $S$, and suppose $C$ not triply connected. There exist two points $p$ and $q$ of $C$ such that $C=A+B$ and $A \cdot B=p+q$, where $A$ and $B$ are closed connected sets neither of which is an arc. Let $r$ be a point of $A-p-q$ and $s$ a point of $B-p-q$. Then on any arc $r x s$ lying in $S-p-q$ there is a last point of $A$ from $r$ to $s$ and a first point of $B$. Let these points be $r$ and $s$ respectively. The open arc $\langle r x s\rangle$ lies in a domain $R$ complementary to $C$ with boundary $J \supset r+s$. Hence this circuit $J$ must pass through $p$ and $q$. But since neither $A$ nor $B$ is an arc there must exist at least two components of $C-J$; one in $A-A \cdot J$ and one in $B-B \cdot J$. Let $N_{1}$ and $N_{2}$ be any two components of $C-J$. Then two points of $\bar{N}_{1} \cdot J$ cannot separate two points of $\bar{N}_{2} \cdot J$ on $J$. For $N_{1}$ and $N_{2}$ are connected sets lying in $S-\bar{R}$ and cannot intersect. If $\bar{N}_{1} \cdot J=\bar{N}_{2} \cdot J=$ three points, then $N_{1}$ and $N_{2}$ must intersect; this is impossible. Therefore, since $C-J$ contains at least two components, $J$ is a split circuit.

Now assume that $C$ contains a split circuit $J$. There is a component $N_{1}$ of $C-J$ with limit points all on the arc $g x h$ of $J$ and a component $N_{2}$ with limit points all on the arc $g y h=J-\langle g x h\rangle$. The arc $g x h$ may be chosen so that $g$ and $h$ are points of $\bar{N}_{1} \cdot J$. Now every component of $C-J$ must have its limit points in either $g x h$ or $g y h$. For suppose some component $N$ of $C-J$ has a limit point $d$ in $\langle g x h\rangle$ and a limit point $e$ in $\langle g y h\rangle$. Every arc of $J$ containing $d$ and $e$ will necessarily contain either $g$ or $h$, limit points of $N_{1}$ which separate $d$ and $e$ on $J$. But this contradicts the fact that $J$ is a split circuit.

Now let $A=g x h+N_{1}$ plus all components of $C-J$ (different from $N_{2}$ ) with limit points on $g x h$, and let $B=g y h+N_{2}$ plus all components of $C-J$ not included in $A$. Then $C=A+B$, where $A$ and $B$ are closed connected subsets of $C$; neither $A$ nor $B$ is a simple arc; and $A \cdot B=g+h$. Therefore $C$ is not triply connected. This completes the proof of Theorem I.

Proof of Theorem II. Suppose $C$ is triply connected. Then every bounding circuit of $C$ has an image which is a c.d.b.* In every map of $C$. For let $C^{\prime}$ be any map of $C$ on a sphere $S^{\prime}$, and $J^{\prime}$ any bounding circuit of $C^{\prime}$. If $C^{\prime}$ does not consist of a simple closed curve, then there is one, and only one, component of $C^{\prime}-J^{\prime}$ (Theorem I), and this component must lie entirely in one of the regions of $S^{\prime}$ bounded by $J^{\prime}$. The other region of $S^{\prime}$ bounded by $J^{\prime}$ must be a complementary domain of $C^{\prime}$. Obviously every c.d.b. of $C^{\prime}$ is also a bounding circuit of $C$.

Therefore if $C^{\prime}$ is a map of $C$ on a sphere $S^{\prime}$ and $C^{\prime \prime}$ a map of $C$ on $S^{\prime \prime}$, every homeomorphism of $C^{\prime}$ into $C^{\prime \prime}$ must preserve complementary domain

* "Complementary domain boundary" will be abbreviated "c.d.b." 
boundaries and is extendable to $S^{\prime}$ and $S^{\prime \prime}$.* Hence $C$ has a unique map on the sphere.

Suppose $C$ is not triply connected. Then $C$ must contain a split circuit $J$ (Theorem I). We shall show first that there is a map of $C$ in which the map of $J$ is a c.d.b. Let $C$ be any map of $C$ on a sphere $S$ and suppose $J$ is not a c.d.b. of $C$. Let $R_{1}$ and $R_{2}$ be the two regions of $S$ bounded by $J$. Since $J$ is not a c.d.b. of $C$ there must exist a component $N_{1}$ of $C-J$ in $R_{1}$ and a component $N_{2}$ in $R_{2}$. Any two points $p$ and $q$ of $\bar{N}_{1} \cdot J$ lie on some c.d.b. of $C$ within $\bar{R}_{2}$. For if every arc from $p$ to $q$ lying in $\bar{R}_{2}$ contains a point of $C$, there would then exist a connected subset of $C$ lying in $R_{\mathbf{2}}$ with limit points on $J$ that separate $p$ and $q . \dagger$ But such a connected subset would belong to a component of $C-J$, and $J$ would not be a split circuit. Hence $p$ and $q$ lie on some c.d.b. within $\bar{R}_{\mathbf{2}}$. Furthermore any three points $p, q, r$ of $\bar{N}_{1} \cdot J$ lie on the same c.d.b. of $C$ within $\bar{R}_{2}$. To show this suppose $p, q, r$ do not lie on the same c.d.b. within $\bar{R}_{\mathbf{2}}$. Then there are three complementary domains of $C$ in $\bar{R}_{\mathbf{2}}$ each containing a pair of $p, q, r$ on its boundary $J_{i},(i=1,2,3)$. Let $l, m, n$ be three arcs, one from each of the boundaries $J_{i}$ such that $l+m+n>p+q+r$ and bounds a region $R_{3}$ which is a subset of $R_{2}$ containing no points of $J_{1}+J_{2}+J_{3}$. This is possible since no two arcs of $l, m, n$ can have a common point. For if two arcs, $l, m$ do have a common point (other than an end point) there would exist a component $N_{3} \supset(l+m)$ of $C-J$ such that $\bar{N}_{3} \cdot J$ contains $p, q, r$, and since $\bar{N}_{1} \cdot J$ contains $p, q, r$, the circuit $J$ would not be a split circuit (definition). Now any two points of $p, q, r$ lie together on the same c.d.b. of $C$ within $\bar{R}_{3}$. For if every arc from $p$ to $q$ (or to $r$ ) lying in $R_{3}$ contained a point of $C$, there would exist, as above, a connected subset of $C$ lying in $\bar{R}_{3}$ and having points on $l+m+n$ that separate $p$ and $q$ on $l+m+n$. There would then exist a component $N_{4}$ of $C-J$ such that $\bar{N}_{4} \cdot J$ contains $p, q$, and $r$; but this is impossible. Now let the circuit $J_{1}$ above be the boundary of a domain $r_{1}$ complementary to $C$ and lying in $R_{2}$. Let $l$ be the arc (of the three $l, m, n$ ) which lies on $J_{1} \supset p+q$. Then since $R_{3}$ contains no points of $J_{1}+J_{2}+J_{3}$, the domain $r_{1}$ is a subset of $R_{2}$, but not of $R_{3}$, and has $p$ and $q$ on its boundary. Let $r_{2}$ be a domain complementary to $C$ which is a subset of $R_{3}$ and has $p$ and $q$ on its boundary. Now $R_{3}$ is not a complementary domain of $C$ since we are assuming that $p, q, r$

* V. W. Adkisson, Cyclicly connected continuous curves whose complementary domain boundaries are homeomorphic, preserving branch points, Comptes Rendus des Séances de la Société des Sciences et des Lettres de Varsovie, Class 3, vol. 23 (1930), p. 167, Theorem 2. If $M$ is a cyclicly connected continuous curve lying on a sphere $S$, and $T$ is a continuous (1-1) correspondence such that $T(M)=M$, a necessary and sufficient condition that $T$ be extendable to $S$ is that for every boundary $J$ of a complementary domain of $M, T(J)$ be also the boundary of a complementary domain of $M$.

$\dagger$ C. Kuratowski, Sur le probleme des courbes gauches en topologie, Fundamenta Mathematicae, vol. 15 (1930), p. 274, Lemma III'. 
do not all lie on the same c.d.b. in $\bar{R}_{2}$. The above process can then be repeated and a third complementary domain $r_{3}$ obtained which is different from $r_{1}$ and $r_{2}$ but also has $p$ and $q$ on its boundary. This process may be continued indefinitely. Hence there exists an infinite number of domains $r_{1}, r_{2}, r_{3}, \ldots$ each complementary to $C$ and having $\mathrm{p}$ and $q$ on its boundary. But this is impossible since there is at most a finite number of complementary domains of $C$ of diameter greater than any $\epsilon>0,{ }^{*}$ and the diameter of each $r_{i}$ is equal to or greater than the distance between $p$ and $q$. We conclude that there is a c.d.b. $L$ within $\bar{R}_{2}$ containing $p, q, r$.

Now any fourth point $s$ of $\bar{N}_{1} \cdot J$ must also lie on $L$. For $s$ and one of the three points $p, q, r$, say $r$, must separate the other two, $p$ and $q$, on $J$. Since $r$ and $s$ must lie on the same c.d.b. of $C$ in $\bar{R}_{\mathbf{2}}$ there exists an arc $\langle r s\rangle$ in $R_{\mathbf{2}}$ such that $\langle r s\rangle \cdot C=0$, and in like manner an arc $\langle p q\rangle$ in $R_{2}$ such that $\langle p q\rangle \cdot C=0$. But $\langle r s\rangle$ and $\langle p q\rangle$ must then have a common point and hence lie in a common complementary domain of $C$. Therefore $p, q, r$, and $s$ all lie on $L$, and all points of $\bar{N}_{1} \cdot J$ must lie on $L$.

Let $D_{1}$ be the complementary domain of $C$ bounded by $L$, and let $(N)$ represent the set of all components $N_{i}$ of $C-J$ in $R_{1}$ for which $\bar{N}_{i} \cdot J=\bar{N}_{i} \cdot L$. Let $\left(N^{\prime}\right)$ be a topological image of $(N)$ in $D_{1}$ such that $C-(N)+\left(N^{\prime}\right)$ is a map of $C$. This is always possible since obviously it would be possible if $C$ were mapped so that $L$ is a circle. This process can be repeated on the map $C-(N)+\left(N^{\prime}\right)$ so that finally a map $C^{\prime}$ is obtained in which $R_{1}$ is a complementary domain of $C^{\prime}$. It follows by well known methods in analysis situs that $C^{\prime}$ is a topological map of $C$. For if the above process is necessary an infinite number of times, $\uparrow$ it involves complementary domains $D_{1}, D_{2}, D_{3}, \cdots$ of $C$ of which only a finite number are of diameter greater than any $\epsilon>0$.

By the same method as above it is possible to map $C$ so that the image of the split circuit $J$ is not a c.d.b. of the image of $C$ since there are at least two components of $C-J$.

Now let $C^{\prime}$ be a map of $C$ on $S^{\prime}$ in which the image of $J$ is a c.d.b. of $C^{\prime}$ and $C^{\prime \prime}$ a map on $S^{\prime \prime}$ in which the image of $J$ is not a c.d.b. of $C^{\prime \prime}$. Then every homeomorphism of $C^{\prime}$ into $C^{\prime \prime}$ is not extendable to $S^{\prime}$ and $S^{\prime \prime}$ since complementary domain boundaries are not preserved. Hence $C$ does not have a unique map, and the theorem is proved.

Corollaries 1 and 2 follow directly from the preceding proof.

* Schoenflies proved (1908) that the complementary domains of a continuous curve are countable, and that at most a finite number have diameters greater than any $\epsilon>0$. See R. L. Moore, Report on continuous curves from the viewpoint of analysis situs, Bulletin of the American Mathematical Society, vol. 29 (1923), pp. 290, 295.

$\dagger$ The components $N_{i}$ are countable. See R. L. Wilder, Concerning continuous curves, Fundamenta Mathematicae, vol. 7 (1925), p. 360, Theorem 9. 
Proof of Theorem III. We shall assume that $M$ has a split point and at the same time a unique map and show that this leads to a contradiction.

Let $M$ be a map on the sphere $S, p$ a split point of $M$, and $A$ and $B$ two closed connected subsets of $M$ such that $M=A+B, A \cdot B=p$, and such that if $A$ or $B$ is an arc, $p$ is not an end point of this arc. Let $R$ be a complementary domain of $M$ whose boundary contains points of both $A$ and $B$. Let $x \neq p$ be a point of $A$ in the boundary of $R$ and $z \neq p$ a point of $B$ in the boundary of $R$. Let $\langle x y z\rangle$ be an arc in $R, x p z$ an $\operatorname{arc}$ of $M, A \supset \operatorname{arc} x p$, and $B \supset \operatorname{arc} p z$. Let $A_{1}$ be a connected component of $A-x p$ such that $\bar{A}_{1}$ has a point $r$ on $x p-x$, and $B_{1}$ a connected component of $B-p z$ such that $\bar{B}_{1}$ has a point $s$ on $p z-z$. Since neither $A$ nor $B$ is a simple arc with end point at $p$, it is possible to choose $x$ and $z$ so that this latter condition may be satisfied. There are two cases to consider.

Case 1. $r \neq s$. Let $M^{\prime}$ represent a map of $M$ on the sphere $S^{\prime}$. Let $T$ be a homeomorphism such that $T(M)=M^{\prime}$ and $T(S)=S^{\prime}$. This is possible since we assume that $M$ has a unique map. Throughout this proof a primed set will indicate the topological image under $T$ of the unprimed set. Now either the set $A_{1}$ lies in the region $D_{1}$ of $S$ bounded by the simple closed curve $C=x p z y x$ while $B_{1}$ lies in the other region $D_{2}$ bounded by $C$, or $A_{1}$ and $B_{1}$ lie in the same region, say $D_{1}$. We shall assume the latter, but the proof is practically the same in either case.

Since $T$ is extendable to $S$ and $S^{\prime}$, the sets $A_{1}^{\prime}$ and $B_{1}^{\prime}$ lie in $D_{1}^{\prime}$. We now construct a new map $M^{\prime \prime}$ of $M$ on $S^{\prime}$ as follows: Let $H_{i}^{\prime},(i=1,2)$, be the subset of $A^{\prime}-\operatorname{arc} x^{\prime} p^{\prime}$ that lies on $D_{i}^{\prime}$. The set $H_{1}^{\prime}$ includes $A_{1}^{\prime}$. Let $H_{1}^{\prime \prime}$ be a topological image of $H_{1}^{\prime}$ lying in $D_{2}^{\prime}-B^{\prime} \cdot D_{2}^{\prime}$, and $H_{2}^{\prime \prime}$ a topological image of $H_{2}^{\prime}$ lying in $D_{1}^{\prime}-B^{\prime} \cdot D_{1}^{\prime}$ such that $x^{\prime} p^{\prime}+H_{1}^{\prime \prime}+H_{2}^{\prime \prime}$ is a topological image of $A^{\prime}$. Then $M^{\prime \prime}=x^{\prime} p^{\prime}+H_{1}^{\prime \prime}+H_{2}^{\prime \prime}+B^{\prime}$. Let $U$ be a homeomorphism such that $U(M)=M^{\prime \prime}$ and $U(S)=S^{\prime}$. Let $U(x y z)=x^{\prime} y^{\prime \prime} z^{\prime}, U\left(H_{i}\right)=H_{i}^{\prime \prime}$, and for points in $B+x p z$ let $U=T$. Since $U$ is extendable, $U\left(D_{1}\right)=D_{1}^{\prime \prime}$ is a region of $S^{\prime}$ containing $U\left(M \cdot D_{1}\right)$. Let $f$ be any arc of $S^{\prime}$ from a point of $A_{1}^{\prime \prime}$ (the topological image of $A_{1}^{\prime}$ in $\left.H_{1}^{\prime \prime}\right)$ to a point of $B_{1}^{\prime}$ which lies entirely in $D_{1}^{\prime \prime}$ including end points. Such an arc must intersect $C^{\prime}$, the boundary of $D_{1}^{\prime}$, since $B_{1}^{\prime}$ lies in $D_{1}^{\prime}$ and $A_{1}^{\prime \prime}$ lies outside $D_{1}^{\prime}$; and since $x^{\prime} p^{\prime} z^{\prime}$ is in the boundary of $D_{1}^{\prime \prime}$ the arc $f$ must intersect $\left\langle x^{\prime} y^{\prime} z^{\prime}\right\rangle$. Then any arc $g$ from $r^{\prime}$ to $s^{\prime}$ lying, except for end points, in $D_{1}^{\prime \prime}$ must intersect $\left\langle x^{\prime} y^{\prime} z^{\prime}\right\rangle$. To show this let $d$ and $e$ be regions about $r^{\prime}$ and $s^{\prime}$ respectively, that do not contain points of $x^{\prime} y^{\prime} z^{\prime}$. It is possible to obtain an arc $t$ that lies entirely in $d$ and joins a point of $g-r^{\prime}$ to a point of $A_{1}^{\prime \prime}$. In like manner we obtain an arc $u$ in $e$ joining a point of $g-s^{\prime}$ to a point of $B_{1}^{\prime}$. The arcs $t$ and $u$ plus the proper subset of $g$ then yield an arc $h$ from $A_{1}^{\prime \prime}$ to $B_{1}^{\prime}$ lying entirely in $D_{1}^{\prime \prime}$. But $h$ must then intersect $\left\langle x^{\prime} y^{\prime} z^{\prime}\right\rangle$, and 
since neither $t$ nor $u$ can intersect $\left\langle x^{\prime} y^{\prime} z^{\prime}\right\rangle$ the arc $g$ must have a point in common with $\left\langle x^{\prime} y^{\prime} z^{\prime}\right\rangle$. Hence there must be a connected subset of $x^{\prime} y^{\prime} z^{\prime}$ lying in $D_{1}^{\prime \prime}$ with two points on $C^{\prime \prime}=x^{\prime} y^{\prime \prime} z^{\prime} p^{\prime} x^{\prime}$ (the boundary of $D_{1}^{\prime \prime}$ ) that separate $r^{\prime}$ and $s^{\prime}$ on $C^{\prime \prime}$. One of these points must obviously lie on the $\operatorname{arc} r^{\prime} s^{\prime}$ of $C^{\prime \prime}$ where $r^{\prime} s^{\prime} \subset\left\langle x^{\prime} p^{\prime} z^{\prime}\right\rangle$. But this is impossible since $\left\langle x^{\prime} p^{\prime} z^{\prime}\right\rangle$ and $\left\langle x^{\prime} y^{\prime} z^{\prime}\right\rangle$ have no common points. Therefore the assumption that every homeomorphism of $M$ is extendable has led to a contradiction, and we conclude that $M$ has no unique map.

Case 2. $r=s=p$. We use the same notation as in Case 1 and obtain in the same manner the map $M^{\prime \prime}$. Any arc joining a point of $A_{1}^{\prime \prime}$ to a point of $B_{1}^{\prime}$ and lying entirely in $D_{1}^{\prime \prime}$ must intersect $\left\langle x^{\prime} y^{\prime} z^{\prime}\right\rangle$. Let $d$ be a region about $p^{\prime}$ that contains no points of $x^{\prime} y^{\prime} z^{\prime}$. Let $f$ be an arc from $A_{1}^{\prime \prime}$ to $B_{1}^{\prime}$ lying in $D_{1}^{\prime \prime}$ and also in $d$. Then $f$ cannot intersect $\left\langle x^{\prime} y^{\prime} z^{\prime}\right\rangle$ but must intersect $\left\langle x^{\prime} y^{\prime} z^{\prime}\right\rangle$. This contradiction shows that $M$ has no unique map and completes the proof of Theorem III.

Proof of Theorem IV. First, assume that $M$ has a unique map. If $M$ is acyclic it must consist of either a simple continuous arc or a single triod since $M$ contains no split point (Theorem III). Obviously these are the only two acyclic peanian continua without split-points.

If $M$ is not acyclic it contains at least one cyclic element $C$ which is a maximal cyclic curve of $M$. Since $M$ cannot contain a split-point there is only one such cyclic element $C$. For if there were a second cyclic element $C_{1}$ which is a maximal cyclic curve of $M$, there would exist a cut-point $p$ of $M$ separating $C$ and $C_{1}, \dagger$ and obviously $p$ would also be a split-point.

If $M-C$ contains a maximal connected acyclic subset with a branch-point $p$, or if $M-C$ contains two arcs with a common end point $p$ on $C$, then $p$ is in either case a split-point. Therefore, $M-C$ contains at most a countable number of simple arcs $\ddagger$ with distinct end points on $C$.

We shall now assume that $C$ is not triply connected and obtain a contradiction of the assumption that $M$ has a unique map. If $C$ is not triply connected, $C$ contains a split circuit $J$ (Theorem I). Let $M$ be a map of $M$ on the sphere $S$ and assume that the components $N_{1}$ and $N_{2}$ of $C-J$ lie in the regions $R_{1}$ and $R_{2}$ of $S$ bounded by $J$. There exists a c.d.b. $L$ of $C$ lying in $\bar{R}_{2}$ such that $\bar{N}_{1} \cdot J=\bar{N}_{1} \cdot L$ (this was shown in the proof of Theorem II). Let $D$ be the complementary domain of $C$ bounded by $L$. Let $\sum a_{i}$ be the set of arcs of $M$ lying in $D$ with end points in $\bar{N}_{1} \cdot L$ and $\sum b_{i}$ the set of $\operatorname{arcs}$ of $M \cdot D$ not included in $\sum a_{i}$. We now obtain a new map $M^{\prime}$ of $M$ on $S$ as follows: Let

${ }^{*}$ Kuratowski, loc. cit., Lemma III'.

† See G. T. Whyburn, loc. cit., p. 168.

$\ddagger$ R. L. Wilder, loc. cit., p. 360, Theorem 9. 
(K) represent all arcs of $M-C$ with end points on $N_{1}$, and let $N_{1}^{\prime}$ be a map in $D$ of $N_{1}+(K)$ such that $\bar{N}_{1} \cdot L=\bar{N}_{1}^{\prime} \cdot L$ and $C-N_{1}+N_{1}^{\prime}$ is a topological image of $C+(K)$. Let $D_{1}$ be the complementary domain of $M-\left[N_{1}+(K)\right]$ that contains $N_{1}+(K)$, and let $\sum a_{i}^{\prime}$ be a topological image of $\sum a_{i}$ in $D_{1}$ such that each $\operatorname{arc} \bar{a}_{i}^{\prime}$ has one end point on $L$, and the set of all these end points of $\sum \bar{a}_{i}^{\prime}$ is identical with the set of end points of $\sum \bar{a}_{i}$ on $L$. For each $b_{i}$ we obtain a new arc $b_{i}^{\prime}$ as follows: If $b_{i} \cdot N_{1}^{\prime}=0$, then $b_{i}^{\prime}=b_{i}$. If $b_{i} \cdot N_{1}^{\prime} \neq 0$, let $d_{i}$ be a region about $Q_{i}$ (the end point of $b_{i}$ on $L$ ) containing no point of $N_{1}^{\prime}$. Then $b_{i}^{\prime}$ is taken as a simple arc which is a subset of $b_{i}$ and lies in $d_{i}$ with end point $Q_{i}$. Then $M^{\prime}=M-N_{1}-(K)+N_{1}^{\prime}-\sum a_{i}+\sum a_{i}^{\prime}-\sum b_{i}+\sum b_{i}^{\prime}$ is a topological image of $M$. But every homeomorphism of $M$ into $M^{\prime}$ cannot be extended to $S$ since $L$ is a c.d.b. of $M$ but not a c.d.b. of $M^{\prime}$. Hence $M$ does not have a unique map, and this contradiction leads to the conclusion that $C$ must be triply connected.

Now suppose $C$ does not consist of a simple closed curve, and there is an arc $b$ in $M-C$ with end point $p$ on two bounding circuits, $J$ and $L$, of $C$. Let $(M-b)$ be any map of $(M-b)$ on $S$. Then $J$ and $L$ bound two complementary domains of $C$ and are outer boundaries of two complementary domains $D_{1}$ and $D_{2}$ of $M-b$. Let $M^{\prime}$ be a map of $M$ obtained by mapping $b$ in $D_{1}$ with end point at $p$, and $M^{\prime \prime}$ a map of $M$ obtained by mapping $b$ in $D_{2}$ with end point at $p$. We have now two essentially different maps of $M$. Hence the end points of the arcs in $M-C$ that lie in $C$ must lie in one, and only one, bounding circuit of $C$.

If $C$ consists of a simple closed curve, $M-C$ cannot contain more than one arc. The proof of this statement is not difficult and will be omitted.

If $M$ consists of a simple continuous arc, the sufficiency of condition (1) follows from the fact that any homeomorphism between two arcs is extendable to a homeomorphism of their planes.* If $M$ consists of a simple closed curve plus an arc, the proof that $M$ has a unique map is easily obtained from the Schoenflies theorem that any homeomorphism between two simple closed curves can be extended to a homeomorphism of their planes.

The proof that a triod has a unique map may also be obtained by a simple application of the Schoenflies theorem.

Suppose $C$ is not a simple closed curve. Let $M$ be a map of $M$ on $S$, and $M^{\prime}$ a map on $S^{\prime}$. The map of $C$ is unique (Theorem II), and from Corollary 1 we see that a c.d.b. of $C$ in one map has an image which is a c.d.b. in every map of $C$. Let $\sum a_{i}$ be the arcs of $M-C$ with end points on the same bounding cir-

${ }^{*}$ R. L. Moore, Conditions under which one of two given closed linear point sets may be thrown into the other one by a continuous transformation of a plane into itself, American Journal of Mathematics., vol. 48 (1926), p. 67. 
cuit $J$ of $C$. Then in the map of $C$ on $S$ these arcs lie in the same complementary domain $D$ of $C$ bounded by $J$, and the map $\sum a_{i}^{\prime}$ of $\sum a_{i}$ on $S^{\prime}$ must lie in the complementary domain $D^{\prime}$ of $C^{\prime}$ bounded by $J^{\prime}$ (the image of $J$ ) since the end points of $\sum \bar{a}_{i}^{\prime}$ lie on $J^{\prime}$ and on no other bounding circuit of $C^{\prime}$.

Now any homeomorphism $T(M)=M^{\prime}$ can be extended to $D$ and $D^{\prime}$. Let $p q$ be one of the $\operatorname{arcs} \bar{a}_{i}$ in $\bar{D}$ with end point $p$ on $J$ such that either $p q$ or its image $p^{\prime} q^{\prime}$ on $S^{\prime}$. is of diameter greater than some $\epsilon>0$. Let $\langle q x y\rangle$ be an arc in $D-\sum a_{i}$ where $y$ is a point of $J$ but not an end point of some arc $a_{i}$ in $D$. Let $\left\langle q^{\prime} x^{\prime} y^{\prime}\right\rangle$ be an $\operatorname{arc}$ in $D^{\prime}-\sum a_{i}^{\prime}$ where $y^{\prime}=T(y)$. Let $r$ and $s$ be points of $J$ that separate $p$ and $y$, and let $r^{\prime}=T(r)$ and $s^{\prime}=T(s)$. Let $a_{k}$ be any arc of $\sum a_{i}$ that lies in the subset $d$ of $D$ bounded by pqxyrp. Then $a_{k}^{\prime}$ must lie in the subset $d^{\prime}$ of $D^{\prime}$ bounded by $p^{\prime} q^{\prime} x^{\prime} y^{\prime} r^{\prime} p^{\prime}$. For if $a_{k}^{\prime}$ lies in $D^{\prime}-\bar{d}^{\prime}$, it must have an end point at either $p^{\prime}$ or $y^{\prime}$. But this is impossible since $y$ was selected not to be an end point, and $p$ cannot be an end point common to two $\operatorname{arcs}$ in $D$. Hence if $\sum b_{i}$ is the subset of $\sum a_{i}$ lying in $d$, then $T\left(\sum b_{i}\right)$ is the subset of $\sum a_{i}^{\prime}$ lying in $d^{\prime}$. The same would be true of any similar subdivision of $D$ and $D^{\prime}$. In fact sides are preserved under $T$ as used by Gehman.* Therefore $T$ can be extended to $D$ and $D^{\prime}$, and in like manner to each complementary domain of $C$ and $C^{\prime}$, and finally to $S$ and $S^{\prime}$. The proof would necessarily be a partial duplication of Gehman's proof and is omitted. This completes the proof of Theorem IV.

Proof of Theorem V. Cases (1), (2), (3) of Theorem V follow easily from Theorem IV.

If $M$ has a unique map and is not acyclic or not a simple closed curve, Theorem III shows that $M$ contains one, and only one, cyclic element $C$ which is a maximal connected cyclic curve of $M$. Furthermore $C$ cannot contain two distinct bounding circuits or consist of a single circuit which would be the outer boundary of two complementary domains of $M$ in any map. For if this were true there would be a circuit $J$ which would be the outer boundary of a bounded complementary domain $R$ of $M$ in some map on a plane $S$ (Corollary 1). Now let $M^{\prime}$ be a map of $M$ on a plane $S^{\prime}$ in which the image of the boundary of $R$ is the boundary of the unbounded complementary domain $R^{\prime}$ of $M^{\prime}$. Then obviously any homeomorphism of $M$ into $M^{\prime}$ which carries the boundary of $R$ into the boundary of $R^{\prime}$ cannot be extended to $R$ and $R^{\prime}$. Therefore $C$ contains only one bounding circuit, and Claytor's result (Corollary (C), p. 810) shows that $C$ consists of a closed 2-cell.

Since $M$ cannot contain a split point, $M-C$ can consist of at most a count-

* H. M. Gehman, On extending a continuous (1-1) correspondence of two plane continuous curves to a correspondence of their planes, these Transactions, vol. 28 (1926), proof of Theorem I, pp. 256-260. 
able number of distinct arcs with distinct end points on the bounding circuit of $C$.

Now suppose $M$ consists of the curve (4) in the theorem. The arcs $M-C$ must all lie in the unbounded complementary domain of $C$ in any map of $M$ in the plane. Let $D$ and $D^{\prime}$ be the two unbounded complementary domains of two maps of $M$ and $M^{\prime}$ respectively. Then any homeomorphism of $M$ into $M^{\prime}$ can be extended to $D$ and $D^{\prime}$ as indicated in the last paragraph of the proof of Theorem IV.

A point $p$ of a peanian continuum $M$ is said to be a local cut-point of $M$ if, and only if, $p$ is a cut-point of some connected open subset of $M^{*}$

The following theorem will be stated without proof:

THEOREM VI. If $M$ is a peanian continuum in a plane $S$, any non-cut-point $p$ of $M$ lies on two (or more) complementary domain boundaries of $M$ if, and only if, $p$ is a local cut-point of $M$. $\dagger$

Now if $M$ is a plane peanian continuum and $C$ a maximal cyclic curve of $M$, every point in $C \cdot(M-C)$ must lie on a bounding circuit of $C$. Since $C$ contains no cut-point (of $C$ ) we can make the following alternative statement of Theorem IV:

The plane peanian continuum $M$ has a unique map on the sphere if, and only if, one of the following conditions holds:

(1) $M$ is acyclic and consists of either a simple arc or a triod,

(2) $M$ contains one cyclic element $C$ which is a maximal triply connected cyclic curve of $M$, and $M-C$ consists of at most a countable number of arcs, $a_{1}, a_{2}, a_{3}, \cdots$, such that $\bar{a}_{i} \cdot \bar{a}_{j}=0,(i \neq j)$, and each $\bar{a}_{i} \cdot C$ is a non-local separating point of $C$, provided that if $C$ is a simple closed curve, then $M-C$ is at most a simple arc.

* See G. T. Whyburn, Local separating points of continua, Monatshefte für Mathematik und Physik, vol. 36 (1929), pp. 305-314.

† This theorem is well known to topologists although the author has been unable to find it stated explicitly in any published paper. See, however, G. T. Whyburn, Local separating points of continua, loc. cit., Theorem 6, and G. T. Whyburn, Concerning points of continuous curves defined by certain im kleinen properties, Mathematische Annalen, vol. 102 (1929), pp. 313-336, Theorem 31.

UNIVERSity OF ARKANSAS,

Fayetreville, Ark. 